\title{
Nonuniqueness and derivative discontinuities in density-functional theories for current-carrying and superconducting systems
}

\author{
K. Capelle \\ Instituto de Química de São Carlos, Universidade de São Paulo, Caixa Postal 780, São Carlos, 13560-970 SP, Brazil \\ G. Vignale \\ Department of Physics and Astronomy, University of Missouri-Columbia, Columbia, Missouri 65211
}

(Received 21 September 2001; published 27 February 2002)

\begin{abstract}
Current-carrying and superconducting systems can be treated within density-functional theory if suitable additional density variables (the current density and the superconducting order parameter, respectively) are included in the density-functional formalism. Here we show that the corresponding conjugate potentials (vector and pair potentials, respectively) are not uniquely determined by the densities. The Hohenberg-Kohn theorem of these generalized density-functional theories is thus weaker than the original one. We give explicit examples and explore some consequences.
\end{abstract}

DOI: 10.1103/PhysRevB.65.113106

PACS number(s): 71.15.Mb, 31.15.Ew, 75.20.-g, 74.25.Jb

Today, density-functional theory ${ }^{1}$ (DFT) is an indispensable tool for the investigation of the electronic structure of matter in atomic, molecular, or extended systems. The theory rests on the celebrated Hohenberg-Kohn (HK) theorem, ${ }^{2}$ which guarantees that the $(v$-representable) ground-state density $n(\mathbf{r})$ uniquely determines the ground-state manybody wave function $\psi_{0}\left(\mathbf{r}_{1}, \ldots, \mathbf{r}_{N}\right)$. This theorem on its own is a very powerful result, but in the original formulation ${ }^{2,3}$ of DFT one can prove even more: the external potential $v(\mathbf{r})$ (e.g., the nuclear charge distribution in a molecule or a solid), too, is a functional of the density, and is unique up to an additive constant. Since this external potential in turn determines all eigenstates of the many-body Hamiltonian, this implies that all observables (and not only ground state ones) are functionals of the ground-state density.

Following original ideas of von Barth and Hedin, ${ }^{4}$ it has recently been shown by Eschrig and Pickett ${ }^{5}$ and by the present authors ${ }^{6}$ that in spin-DFT (SDFT) the situation is not that simple: while the wave function is still uniquely determined by the spin densities $n_{\uparrow}(\mathbf{r})$ and $n_{\downarrow}(\mathbf{r})$, the external potentials $v_{\uparrow}(\mathbf{r})$ and $v_{\downarrow}(\mathbf{r})$ [or $v(\mathbf{r})$ and $\left.\mathbf{B}(\mathbf{r})\right]$ are not. This implies that SDFT functionals are not always differentiable, and has far-reaching consequences for the construction of better exchange-correlation (XC) functionals, and for applications to systems such as half-metallic ferromagnets. ${ }^{5,6}$

SDFT is not the only instance at which the original HK theorem has been generalized. In the present work we extend the analysis of Ref. 6 to two other generalizations of DFT, namely, current-DFT ${ }^{7,8}$ (CDFT) and DFT for superconductors. ${ }^{9-12}$ The discovery of nonuniqueness in these generalized DFTs deepens our understanding of the respective XC functionals and flags a warning signal to alltoo-immediate generalizations of the original $\mathrm{HK}$ theorem to more complex situations.

The basic physics of nonuniqueness is simple. When a sufficiently small change in one of the external fields does not change the corresponding density distribution, the associated susceptibility vanishes. The search for, and the interpretation of, nonuniqueness in DFT is thus guided by inves- tigations of the circumstances under which some response function becomes zero.

We first consider current-carrying systems. The appropriate formulation of (nonrelativistic) DFT is $\mathrm{CDFT}^{7,8}$ which is based on the many-body Hamiltonian (in atomic units, i.e., $\hbar=e=m=1)$

$$
\begin{aligned}
\hat{H}= & \hat{T}+\hat{U}+\int d^{3} r \hat{n}(\mathbf{r})[v(\mathbf{r})-\mu]+\frac{1}{c} \int d^{3} r \hat{\mathbf{j}}_{p}(\mathbf{r}) \mathbf{A}(\mathbf{r}) \\
& +\frac{1}{2 c^{2}} \int d^{3} r \hat{n}(\mathbf{r}) A^{2}(\mathbf{r})+\int d^{3} r \hat{\mathbf{m}}(\mathbf{r}) \mathbf{B}(\mathbf{r}),
\end{aligned}
$$

where $\mathbf{B}(\mathbf{r})=\boldsymbol{\nabla} \times \mathbf{A}(\mathbf{r})$ is the magnetic field, $v(\mathbf{r})$ the electrostatic one, and $\hat{T}$ and $\hat{U}$ denote the operators for kineticenergy and particle-particle interaction, respectively.

The basic variables of CDFT, in terms of which the entire ground-state physics of the current-carrying many-body system is described, are $n(\mathbf{r}), \mathbf{m}(\mathbf{r})$, and $\mathbf{j}_{p}(\mathbf{r})$, the ground-state expectation values of the particle-density operator $\hat{n}(\mathbf{r})$ $=\Sigma_{\sigma} \Psi_{\sigma}^{\dagger}(\mathbf{r}) \Psi_{\sigma}(\mathbf{r}), \quad$ spin-magnetization operator $\hat{\mathbf{m}}(\mathbf{r})$ $=(1 / 2 c) \Sigma_{\alpha, \beta} \Psi_{\alpha}(\mathbf{r}) \hat{\boldsymbol{\sigma}} \Psi_{\beta}(\mathbf{r})$, and (paramagnetic) currentdensity operator

$$
\hat{\mathbf{j}}_{p}(\mathbf{r})=\frac{1}{2 i} \sum_{\sigma}\left\{\Psi_{\sigma}^{\dagger}(\mathbf{r})\left[\nabla \Psi_{\sigma}(\mathbf{r})\right]-\left[\nabla \Psi_{\sigma}^{\dagger}(\mathbf{r})\right] \Psi_{\sigma}(\mathbf{r})\right\},
$$

where the $\Psi_{\sigma}(\mathbf{r})$ are field operators and $\hat{\boldsymbol{\sigma}}$ is the vector of Pauli matrices.

According to the CDFT version of the HK theorem these densities uniquely determine the ground-state many-body wave function. However, in striking contrast to conventional "density only" DFT they do not uniquely determine the potentials $\mathbf{A}(\mathbf{r}), \mathbf{B}(\mathbf{r})$, and $v(\mathbf{r})$ : it is possible to find different vector and scalar potentials that yield the same ground state, and consequently the same densities $n(\mathbf{r}), \mathbf{m}(\mathbf{r})$, and $\mathbf{j}_{p}(\mathbf{r})$.

Before delving into a general characterization of such potentials, we present a simple example that clearly displays 
the problem. We consider an atom subjected to a uniform magnetic field $\mathbf{B}=B \hat{\mathbf{z}}$, where $\hat{\mathbf{z}}$ is the unit vector along the $z$ axis. Ignoring spin-orbit interactions, the Hamiltonian can be written as

$$
\hat{H}=\sum_{i}\left(\frac{\hat{p}_{i}^{2}}{2}-\frac{Z}{r_{i}}+\frac{B^{2} r_{\perp, i}^{2}}{8 c^{2}}\right)+\sum_{i \neq j} \frac{1}{2 r_{i j}}+\frac{\hat{L}_{z}+2 \hat{S}_{z}}{2 c} B,
$$

where $r_{\perp, i}^{2} \equiv x_{i}^{2}+y_{i}^{2}$, and $\hat{L}_{z}$ and $\hat{S}_{z}$ are the $z$ components of the orbital and spin angular-momentum operators $\mathbf{L}$ and $\mathbf{S}$. $Z$ is the atomic number, specifying the external potential. Both $\hat{L}_{z}$ and $\hat{S}_{z}$ are constants of motion, hence the (nondegenerate) ground state of $\hat{H}$ is also an eigenstate of $\hat{L}_{z}$ and $\hat{S}_{z}$, with eigenvalues $m_{L}$ and $m_{S}$ respectively. The ground-state energy is $E_{0}$.

Consider now the same system of $Z$ electrons being subjected to a different (but still uniform) magnetic field $\mathbf{B}^{\prime}$ $=B^{\prime} \hat{\mathbf{z}}$ and the external potential

$$
v^{\prime}(r)=-\frac{Z}{r}-\frac{1}{8 c^{2}}\left(B^{\prime 2}-B^{2}\right) r_{\perp}^{2} .
$$

The Hamiltonian of this system is

$$
\hat{H}^{\prime}=\hat{H}+\frac{1}{2 c}\left(\hat{L}_{z}+2 \hat{S}_{z}\right)\left(B^{\prime}-B\right) .
$$

Thus, we immediately see that the ground state of $\hat{H}$ (or, for that matter, any simultaneous eigenstate of $\hat{H}, \hat{L}_{z}$, and $\left.\hat{S}_{z}\right)$ is also an eigenstate of $\hat{H}^{\prime}$ with eigenvalue $E^{\prime}=E_{0}$ $+(1 / 2 c)\left(m_{L}+2 m_{S}\right)\left(B^{\prime}-B\right)$. Furthermore, if the difference $B^{\prime}-B$ is not too large, this eigenstate will be the ground state of $\hat{H}^{\prime}$; the qualitative condition for this to happen is that $E^{\prime}-E_{0} \ll E_{G}$ where $E_{G}$ is the energy gap between the first excited state and the ground state of $\hat{H}$. Thus, we have succeeded in constructing two different sets of potentials, $\mathbf{A}$ $=(\mathbf{B} \times \mathbf{r}) / 2$ and $v$, and $\mathbf{A}^{\prime}=\left(\mathbf{B}^{\prime} \times \mathbf{r}\right) / 2$ and $v^{\prime}$, that yield the same ground state.

Let us now consider the question from a more general point of view. Let $\mathbf{A}^{\prime}=\mathbf{A}+\Delta \mathbf{A}$ and $v^{\prime}=v+\Delta v$ be vector and scalar potentials that are supposed to yield the same ground state $\psi_{0}$ as $\mathbf{A}$ and $v$. A necessary condition for this is that $\psi_{0}$ satisfy the eigenvalue equation

$$
\int d^{3} r\left[\hat{n} \Delta v+\frac{1}{2 c^{2}} \hat{n} \Delta \mathbf{A}^{2}+\frac{1}{c} \hat{\mathbf{j}}_{p} \Delta \mathbf{A}\right] \psi_{0}=\Delta E \psi_{0},
$$

where we neglected, for simplicity, the spin degrees of freedom, because the nonuniqueness associated with them is already discussed in Refs. 5 and 6.

The general problem at hand is thus to find a linear combination of the density operators $\hat{n}(\mathbf{r})$ and $\hat{\mathbf{j}}_{p}(\mathbf{r})$ that has $\psi_{0}$ as eigenfunction. This problem is not easily solved in general. It is easy, however, to obtain a particular solution of Eq. (6) if one can find a linear combination of the density operators that is a constant of motion. The ground state of $\hat{H}$ is automatically an eigenstate of such a constant of motion, and
Eq. (6) is satisfied. By making the coefficients of the linear combination sufficiently small we can always ensure that $\psi_{0}$ remains the ground state of the Hamiltonian with the new potentials (assuming of course that the spectrum of $\hat{H}$ has a gap between its ground state and first excited state). This is the same prescription employed in Ref. 6 to construct examples for nonuniqueness in SDFT. In the terminology of that reference nonuniqueness arising from such constants of motion is referred to as systematic nonuniqueness.

As a trivial example of this procedure consider the constant of motion $\hat{N}=\int d^{3} r \hat{n}(\mathbf{r})$. The existence of this constant of motion tells us that $\Delta v(\mathbf{r})=$ const, $\Delta \mathbf{A}(\mathbf{r})=0$ is a solution of Eq. (6). This is the well-known nonuniqueness of the scalar potential with respect to the addition of a constant. Consider now the less trivial example

$$
\hat{L}_{z}=\int d^{3} r(\hat{\mathbf{z}} \times \mathbf{r}) \cdot \hat{\mathbf{j}}_{p}(\mathbf{r}),
$$

which is a constant of motion in any system that is invariant under rotations about the $z$ axis. Comparing this with Eq. (6) we immediately see that $\Delta \mathbf{A}(\mathbf{r})=\Delta B(\hat{\mathbf{z}} \times \mathbf{r}) / 2$ and $\Delta v(\mathbf{r})$ $=-[\Delta \mathbf{A}(\mathbf{r})]^{2} /\left(2 c^{2}\right)$ with $\Delta B=$ const is indeed a solution of the posed problem. This is, of course, nothing but a more formal derivation of the elementary example discussed above.

Another way in which nonuniqueness can arise is by adding an operator to the Hamiltonian that, although not a constant of motion, happens to have eigenvalue zero on the ground state. This was called accidental nonuniqueness in Ref. 6. To give an example in CDFT, let $n_{\sigma}(\mathbf{r})$ and $\mathbf{j}_{p \sigma}(\mathbf{r})$ denote the exact spin-resolved ground-state density and paramagnetic current of a two-electron system, such as the $\mathrm{He}$ atom, in the presence of external vector and scalar potentials. For sufficiently small external fields these densities must arise from the single-particle orbitals $\varphi_{\uparrow}(\mathbf{r})$ and $\varphi_{l}(\mathbf{r})$ that are the lowest-energy solutions of the spin-dependent KohnSham (KS) equations

$$
\left\{\frac{1}{2}\left(-i \boldsymbol{\nabla}+\frac{1}{c} \mathbf{A}_{s \sigma}(\mathbf{r})\right)^{2}+v_{s \sigma}(\mathbf{r})\right\} \varphi_{\sigma}(\mathbf{r})=\epsilon_{\sigma} \varphi_{\sigma}(\mathbf{r}),
$$

where $\mathbf{A}_{s \sigma}(\mathbf{r})$ and $v_{s \sigma}(\mathbf{r})$ are the KS potentials, defined, as usual, in terms of the external, Hartree, and exchangecorrelation potentials. The relation between the densities and the single-particle orbitals is $n_{\sigma}(\mathbf{r})=\left|\varphi_{\sigma}(\mathbf{r})\right|^{2}$ and

$$
\mathbf{j}_{p \sigma}(\mathbf{r})=n_{\sigma}(\mathbf{r}) \nabla \phi_{\sigma}(\mathbf{r}),
$$

where $\phi_{\sigma}(\mathbf{r})$ is the phase of the complex orbital $\varphi_{\sigma}(\mathbf{r}) .^{13}$

Equation (8) can be rewritten in the form

$$
\left\{\frac{1}{2}\left(-i \boldsymbol{\nabla}+\frac{1}{c}\left[\mathbf{A}_{s \sigma}+c \boldsymbol{\nabla} \phi_{\sigma}\right]\right)^{2}+v_{s \sigma}\right\}\left|\varphi_{\sigma}\right|=\boldsymbol{\epsilon}_{\sigma}\left|\varphi_{\sigma}\right|,
$$

which has the solution $\mathbf{A}_{s \sigma}(\mathbf{r})=-c \boldsymbol{\nabla} \phi_{\sigma}(\mathbf{r})$ $=-c \mathbf{j}_{p \sigma}(\mathbf{r}) / n_{\sigma}(\mathbf{r})$ and 


$$
v_{s \sigma}(\mathbf{r})=\frac{1}{2} \frac{\nabla^{2}\left|\varphi_{\sigma}(\mathbf{r})\right|}{\left|\varphi_{\sigma}(\mathbf{r})\right|}+\epsilon_{\sigma}=\frac{1}{2} \frac{\nabla^{2} n_{\sigma}^{1 / 2}(\mathbf{r})}{n_{\sigma}^{1 / 2}(\mathbf{r})}+\epsilon_{\sigma} .
$$

To determine whether these are the only potentials that reproduce the given densities $n_{\sigma}(\mathbf{r})$ and $\mathbf{j}_{p \sigma}(\mathbf{r})$ we assume the existence of a second such set of potentials, $\mathbf{A}_{s \sigma}^{\prime}(\mathbf{r})$ $=\mathbf{A}_{s \sigma}(\mathbf{r})+\Delta \mathbf{A}_{s \sigma}(\mathbf{r})$ and $v_{s \sigma}(\mathbf{r})=v_{s \sigma}(\mathbf{r})+\Delta v_{s \sigma}(\mathbf{r})$. By substituting these back in Eq. (10) and separating the real and the imaginary parts we obtain

$$
v_{s \sigma}^{\prime}(\mathbf{r})+\frac{1}{2 c^{2}} \Delta \mathbf{A}_{s \sigma}^{2}(\mathbf{r})=\frac{1}{2} \frac{\nabla^{2} n_{\sigma}^{1 / 2}(\mathbf{r})}{n_{\sigma}^{1 / 2}(\mathbf{r})}+\epsilon_{\sigma}
$$

and $\boldsymbol{\nabla} \cdot\left[n_{\sigma}(\mathbf{r}) \Delta \mathbf{A}_{s \sigma}(\mathbf{r})\right]=0$. This last equation follows more directly from the application of the continuity equation to the real solution of Eq. (10). Its general solution is $\Delta \mathbf{A}_{s \sigma}(\mathbf{r})$ $=\boldsymbol{\nabla} \times \mathbf{Q}_{\sigma}(\mathbf{r}) / n_{\sigma}(\mathbf{r})$ where $\mathbf{Q}_{\sigma}(\mathbf{r})$ is an arbitrary vector field. Hence,

$$
v_{s \sigma}^{\prime}(\mathbf{r})=v_{s \sigma}(\mathbf{r})-\frac{1}{2 c^{2}}\left(\frac{\boldsymbol{\nabla} \times \mathbf{Q}_{\sigma}(\mathbf{r})}{n_{\sigma}(\mathbf{r})}\right)^{2}
$$

and

$$
\mathbf{A}_{s \sigma}^{\prime}(\mathbf{r})=\mathbf{A}_{s \sigma}(\mathbf{r})+\frac{\boldsymbol{\nabla} \times \mathbf{Q}_{\sigma}(\mathbf{r})}{n_{\sigma}(\mathbf{r})} .
$$

By construction, $\mathbf{A}_{s \sigma}(\mathbf{r})$ and $v_{s \sigma}(\mathbf{r})$ are the potentials for which $n_{\sigma}(\mathbf{r})$ and $\mathbf{j}_{p \sigma}(\mathbf{r})$ are ground-state densities. If $\mathbf{Q}_{\sigma}$ is sufficiently small and if the KS system at $\mathbf{Q}_{\sigma}=0$ has an energy gap separating the first excited state from the ground state, $\varphi_{\sigma}(\mathbf{r})$ will remain the ground state in the potentials $\mathbf{A}_{s \sigma}^{\prime}(\mathbf{r})$ and $v_{s \sigma}^{\prime}(\mathbf{r})$. Thus, Eqs. (13) and (14) provide a vivid and nontrivial example of nonuniqueness of the KS potentials of CDFT.

Next, we turn to the superconducting case. Here the underlying many-body Hamiltonian is ${ }^{9}$

$$
\begin{aligned}
\hat{H}= & \hat{T}+\hat{U}+\int d^{3} r \hat{n}(\mathbf{r})[v(\mathbf{r})-\mu]+\int d^{3} r \hat{\mathbf{m}}(\mathbf{r}) \mathbf{B}(\mathbf{r}) \\
& -\int d^{3} r \int d^{3} r^{\prime}\left[\hat{\chi}\left(\mathbf{r}, \mathbf{r}^{\prime}\right) D^{*}\left(\mathbf{r}, \mathbf{r}^{\prime}\right)+\text { H.c. }\right],
\end{aligned}
$$

where the expectation value of the pair operator $\hat{\chi}\left(\mathbf{r}, \mathbf{r}^{\prime}\right)$ $=\Psi_{\uparrow}(\mathbf{r}) \Psi_{\downarrow}\left(\mathbf{r}^{\prime}\right)$ is the superconducting order parameter and $D\left(\mathbf{r}, \mathbf{r}^{\prime}\right)$ the corresponding pair potential. The phononinduced interaction term of Ref. 9 can be added to $\hat{H}$ without changing our conclusions.

As above, we now assume that the densities $n(\mathbf{r}), \mathbf{m}(\mathbf{r})$, and $\chi\left(\mathbf{r}, \mathbf{r}^{\prime}\right)$ can also be reproduced in different fields $v^{\prime}$ $=v+\Delta v, \quad \mathbf{B}^{\prime}=\mathbf{B}+\Delta \mathbf{B}$, and $D^{\prime}=D+\Delta D$. The equation obeyed by $\Delta v, \Delta \mathbf{B}$, and $\Delta D$ is

$$
\int d^{3} r\left[\hat{n} \Delta v+\hat{\mathbf{m}} \Delta \mathbf{B}-\int d^{3} r^{\prime}(\hat{\chi} \Delta D+\text { H.c. })\right] \psi_{0}=\Delta E \psi_{0} .
$$

At this stage we already see a first nontrivial difference to the case of CDFT and SDFT: due to the presence of the pair operator $\hat{\chi}$ in $\hat{H}$ the particle number operator $\hat{N}$ is not a constant of motion, and we are not free to add an arbitrary constant to the external potential $v(\mathbf{r})$. In other words $\Delta D$ $=0, \Delta \mathbf{B}=0$, and $\Delta v=$ const is not a solution of Eq. (16) for a given $\psi_{0}$. DFT for superconductors thus does not suffer from the most basic nonuniqueness of all, that with respect to the additive constant in the electrostatic potential.

However, DFT for superconductors is not free of nonuniqueness. For a singlet superconductor the spin susceptibility vanishes at zero temperature. ${ }^{14}$ In the light of our physical characterization of nonuniqueness at the beginning of this paper we would thus expect some associated nonuniqueness. Indeed, this is bourne out by more detailed analysis. If $\mathbf{B}=B \hat{\mathbf{z}}$ is spatially uniform and sufficiently weak not to break Cooper pairs paramagnetically, then $\mathbf{B}^{\prime}=\mathbf{B}+\Delta \mathbf{B}$, where $\Delta \mathbf{B}$ is also weak, uniform, and parallel to $\hat{\mathbf{z}}$, has the same ground state, because under these circumstances $\hat{M}_{z}=$ $\int d^{3} r \hat{m}_{z}$ is a conserved quantity, i.e., the superconductor remains in a singlet state, with all electrons paired up. Consequently, the set of potentials $\{v, \mathbf{B}, D\}$ is not uniquely determined by the conjugate densities $\{n, \mathbf{m}, \chi\}$. Since it is associated with the constant of motion $\hat{M}_{z}$, this is systematic nonuniqueness in the above sense.

With these examples we end our list of explicit occurrences of nonuniqueness in generalized DFTs, and now turn to a discussion of broader aspects of our findings.

In early papers on both $\mathrm{CDFT}^{7,8}$ and DFT for superconductors ${ }^{10}$ one finds the statement that the chosen densities uniquely determine the corresponding potentials. As we have shown here, these statements are not accurate, and all that is determined uniquely is the ground-state wave function. Concerning consequences of this finding we refer the reader to the discussion we have given earlier of consequences of nonuniqueness in SDFT. ${ }^{6}$ That discussion carries over almost literally to the case of current-carrying and superconducting systems. However, we wish to stress particularly that for most applications of any DFT, including CDFT and DFT for superconductors, uniqueness of the ground-state wave function is sufficient, since no explicit use of the density-potential relation is made. A notable exception within CDFT is the recent work by Lee and Handy, ${ }^{15}$ in which it is attempted to systematically construct exact CDFT potentials from given densities. This construction must be reexamined in view of our finding that the CDFT potentials are not uniquely determined by the densities. Further exceptions are listed in Ref. 6.

Another important consequence of nonuniqueness in DFT arises from the connection between the external and KS potentials with the functional derivatives of the kinetic- and internal-energy functionals. For CDFT these connections take the form (neglecting, for simplicity, again the spin degrees of freedom)

$$
-\frac{\delta T_{s}\left[n, \mathbf{j}_{p}\right]}{\delta n(\mathbf{r})}=v_{s}(\mathbf{r})-\mu+\frac{1}{2 c} \mathbf{A}_{s}(\mathbf{r})^{2}
$$




$$
-\frac{\delta T_{s}\left[n, \mathbf{j}_{p}\right]}{\delta \mathbf{j}_{p}(\mathbf{r})}=\frac{1}{c} \mathbf{A}_{s}(\mathbf{r}),
$$

i.e., derivatives of the noninteracting kinetic energy $T_{s}$ determine the KS potentials $v_{s}$ and $\mathbf{A}_{s}$. Similarly, the derivatives of the internal-energy functional $F$, defined as the groundstate expectation value of $\hat{T}$ and $\hat{U}$, determine the external potentials $v(\mathbf{r})$ and $\mathbf{A}(\mathbf{r})$. Analogous equations hold also in DFT for superconductors.

From Eqs. (17) and (18) we see that nonuniqueness of the Kohn-Sham potentials implies that the derivatives on the left-hand side do not exist on the space of all densities, for, if they existed, they would determine the potentials uniquely. Consequently, the functionals $T_{s}$ and $F$ display multiple derivative discontinuities and must be redefined on equivalence classes of densities arising from the potentials modulo the nonunique pieces. The same applies to the $\mathrm{XC}$ functional $E_{X C}$ itself, since $E_{X C}$ is in general defined as the difference $E_{X C}=F-T_{s}-E_{H}$, where $E_{H}$ stands for all Hartree-like terms included in the respective formulation of DFT. Common approximations to $T_{S}$ and $E_{X C}$ do not display these derivative discontinuities. Judging from experience ${ }^{16}$ with similar discontinuities in ordinary DFT we expect this shortcoming to be most relevant for the calculation of energy gaps.

The nonuniqueness problem discussed above (as well as the intimately related nondifferentiability problem) occurs, strictly speaking, only at zero temperature. At finite temperature one should work with a statistical ensemble, rather than with a ground state, and then the uniqueness of the relation between density and potential is restored. ${ }^{17}$ However, the singularity at $T=0$ is an indicator that a real physical problem exists. Consider, as an illustration, the nonuniqueness of the potentials of SDFT, discussed in Refs. 5 and 6. Due to the nondifferentiability of the $\mathrm{XC}$ functional, an infinitesimal change in the spin density (such as the change $\delta m$ caused by the flipping of a single electron in an extended half-metallic ferromagnet) may cause a finite (discontinuous) change in the $\mathrm{XC}$ potential. None of the existing approximations is able to reproduce such a discontinuity. Going to finite but small temperatures simply replaces the discontinuity by a very rapid continuous change. To estimate the scale of this change we note that at $T=0$ the magnetic field is only determined by the densities to within $E_{G} / \mu_{0}$, where $E_{G}$ is the energy gap and $\mu_{0}$ the Bohr magneton. Multiplying this with the lowtemperature spin susceptibility we find that the spin density changes by $\left(\delta m E_{G} / k_{B} T\right) \exp \left(-E_{G} / k_{B} T\right)$. As long as $k_{B} T$ $\ll E_{G}$ this is much less than the physically relevant change $\delta m$, and therefore the functional remains effectively discontinuous in the low-temperature regime.

In summary, we have shown that generalizations of DFT to current-carrying and to superconducting systems suffer from the same nonuniqueness problem we earlier discussed for the case of spin-polarized systems. Although the details are interestingly different in each of these three cases, the physical connection of nonuniqueness with a vanishing response function, as well as the classification of nonuniqueness into systematic (arising from constants of motion) and accidental (arising from special features of the ground state), and the consequences for differentiability of the respective density functionals $T_{s}$ and $E_{X C}$ are the same in all three cases, and, we believe, also in any other generalization of DFT.

K.C. thanks the FAPESP for financial support. G.V. acknowledges support from NSF Grant No. DMR-0074959.
${ }^{1}$ W. Kohn, Rev. Mod. Phys. 71, 1253 (1999).

${ }^{2}$ P. Hohenberg and W. Kohn, Phys. Rev. 136, B864 (1964).

${ }^{3}$ W. Kohn and L.J. Sham, Phys. Rev. 140, A1133 (1965).

${ }^{4}$ U. von Barth and L. Hedin, J. Phys. C 5, 1629 (1972).

${ }^{5}$ H. Eschrig and W.E. Pickett, Solid State Commun. 118, 123 (2001).

${ }^{6}$ K. Capelle and G. Vignale, Phys. Rev. Lett. 86, 5546 (2001).

${ }^{7}$ G. Vignale and M. Rasolt, Phys. Rev. Lett. 59, 2360 (1987).

${ }^{8}$ G. Vignale and M. Rasolt, Phys. Rev. B 37, 10685 (1988).

${ }^{9}$ L.N. Oliveira, E.K.U. Gross, and W. Kohn, Phys. Rev. Lett. 60, 2430 (1988).

${ }^{10}$ W. Kohn, E.K.U. Gross, and L.N. Oliveira, J. Phys. (France) 50, 2601 (1989).

${ }^{11}$ S. Kurth, M. Marques, M. Lüders, and E.K.U. Gross, Phys. Rev.
Lett. 83, 2628 (1999)

${ }^{12}$ W.M. Temmermann, Z. Szotek, B.L. Gyorffy, O.K. Andersen, and O. Jepsen, Phys. Rev. Lett. 76, 307 (1996).

${ }^{13}$ Equation (9) implies that $\boldsymbol{\nabla} \times\left(\mathbf{j}_{p \sigma} / n_{\sigma}\right)=0$, i.e., only states of zero vorticity are noninteracting $v$ representable in a twoelectron system.

${ }^{14}$ M. Tinkham, Introduction to Superconductivity, 2nd ed. (McGraw-Hill, New York, 1996).

${ }^{15}$ A.M. Lee and N.C. Handy, Phys. Rev. A 59, 209 (1999).

${ }^{16}$ J.P. Perdew, R.G. Parr, M. Levy, and J.L. Balduz, Phys. Rev. Lett. 49, 1691 (1982); see also J.P. Perdew and M. Levy, ibid. 51, 1884 (1983).

${ }^{17}$ N. Argaman and G. Makov, Am. J. Phys. 68, 69 (2000). 